\title{
BMJ Open Protocol for developing a healthcare transition intervention for young people with spinal cord injuries using a participatory action research approach
}

\author{
Emily Alice Bray (D), Ajesh George (D), Bronwyn Everett (D), \\ Yenna Salamonson (D) , Lucie Ramjan
}

To cite: Bray EA, George A, Everett B, et al. Protocol for developing a healthcare transition intervention for young people with spinal cord injuries using a participatory action research approach. BMJ Open 2021;11:e053212. doi:10.1136/ bmjopen-2021-053212

- Prepublication history and additional supplemental material for this paper are available online. To view these files, please visit the journal online (http://dx.doi.org/10.1136/ bmjopen-2021-053212).

Received 10 May 2021 Accepted 08 July 2021

Check for updates

(C) Author(s) (or their employer(s)) 2021. Re-use permitted under CC BY-NC. No commercial re-use. See rights and permissions. Published by BMJ.

School of Nursing and Midwifery, Western Sydney University, Penrith, New South Wales, Australia

Correspondence to Mrs Emily Alice Bray; 16251104@student. westernsydney.edu.au

\section{ABSTRACT}

Introduction While healthcare transition (HCT)

interventions are recognised as an important area in paediatric rehabilitation, there has been limited research focusing on young people with spinal cord injuries (SCI). In this study, researchers will collaborate with young people with $\mathrm{SCl}$ and their parents/caregivers to develop, implement and evaluate the feasibility and acceptability of a HCT intervention aimed at supporting young people with $\mathrm{SCl}$ during their transition from paediatric to adult healthcare services.

Methods and analysis A participatory action research (PAR) approach will be used to co-develop the HCT intervention with young people with $\mathrm{SCl}$ aged 14-25 years and their parents/caregivers. Three phases will be conducted to address the five objectives of this study. Phase 1 will use semi-structured interviews to explore young people and parent/caregivers' experiences of HCT. In Phase 2a, both young people and parent/caregivers will be co-researchers. They will be included in the analysis of the interviews and will be asked to participate in co-design workshops to inform the development of a prototype HCT intervention. In Phase $2 b$, using focus groups, feedback on the prototype HCT intervention will be collected. In Phase 3, the refined prototype HCT intervention will be implemented, and young people with $\mathrm{SCl}$ and parent/ caregivers will evaluate the feasibility and acceptability of the HCT intervention in semi-structured interviews. A reference group, including stakeholders and end users, will be consulted at different time points.

Ethics and dissemination The study has received ethics approval from Western Sydney University Human Research and Ethics Committee (H14029). The researcher will use the results of this study as chapters in a thesis to obtain a Doctor of Philosophy degree. The findings will be disseminated via publication in peer-reviewed journals and will be presented at local, national or international conferences.

Trial registration number ACTRN12621000500853

\section{INTRODUCTION}

Healthcare transition (HCT) is 'the purposeful, planned movement of adolescents and young adults with chronic physical and medical conditions from child-centred
Strengths and limitations of this study

- This study is the first study to use a participatory action research (PAR) approach and involve youth with spinal cord injuries to co-create and evaluate a healthcare transition (HCT) support intervention.

- Using PAR methodology will give a voice to a group of young people whose voices have historically been ignored.

- Inclusion of PAR principles in the development of the HCT intervention increases the likelihood that the intervention will be acceptable to end users.

- Clear articulation of the methods of the study will provide guidance on the use of PAR in the development of HCT interventions.

- PAR requires prolonged engagement with participants and is time intensive.

to adult-oriented healthcare systems'. ${ }^{1}$ Transition from the paediatric to adult healthcare system is a complex process that requires care to be delivered in a coordinated and uninterrupted manner through the provision of developmentally appropriate and comprehensive services. ${ }^{1-4}$

Challenges in the process of transition occur as a result of the procedural and cultural differences between paediatric and adult healthcare, and the crucial yet turbulent developmental phase of adolescence. ${ }^{4-6}$ Families speak about the difficulty of terminating the lifelong relationships that have developed with paediatric providers, the challenges of building working relationships in new healthcare settings and the fears that adult professionals lack the knowledge and quality of care provided by their paediatric providers. ${ }^{6}$ Furthermore, the adult healthcare system is characterised by decreased family involvement where success managing your health requires skills such as selfadvocacy and self-determination. Yet, there 
is evidence to suggest that developing these skills is not deemed a priority for young people with chronic conditions transitioning out of the paediatric sector. ${ }^{4}$ Adolescence is also a complex phase, with biological maturity preceding psychosocial maturity. This may contribute to tension arising between adolescents, their families and healthcare providers as they attempt to find an identity for themselves outside of the family unit and push the boundaries of these relationships. ${ }^{4}$

The aforementioned challenges associated with the move from paediatric to adult healthcare systems and inadequate transition preparations can alter the health outcomes of young people with chronic conditions. ${ }^{28}$ Research indicates that post transition some young people with chronic conditions often fail to adhere to treatments, are lost to follow-up, experience deteriorating health, develop secondary complications and face negative social and emotional outcomes. ${ }^{27}$ Despite this, there is evidence to suggest that effective HCT interventions can improve health outcomes for young people with chronic conditions. ${ }^{8}$

This study aims to address the transition needs of young people with spinal cord injuries (SCI) and the current gap in services, contributing to the evidence while improving transition outcomes and quality of life for these individuals. SCI is a catastrophic event that impairs conduction of sensory and motor signals across the site(s) of lesion(s), as well as the autonomic nervous system, resulting in physical disability and impaired function of various organ systems. ${ }^{9}$ Paediatric-onset SCI is relatively rare but carries significant psychological and physiological consequences. Impaired mobility and long-term risks for secondary complications including bowel and urinary complications, pressure injury, pain and autonomic dysfunction, can lead to decreased independence, less community participation and negative psychosocial outcomes. ${ }^{10} 11$ Access to coordinated adult healthcare can facilitate the management of long-term risks during the transition to adulthood for young people with SCI. However, little has been written on HCT interventions for young people with a paediatric-onset SCI $^{12}{ }^{13}$ and what does exist suggests that there is a lack of support for young people with SCI transitioning into adulthood and adult healthcare services. ${ }^{13}$

\section{Aims}

The overall aim of this study is to co-develop, implement and evaluate a HCT intervention to support young people with SCI. The specific objectives will be to:

1. Identify current services and resources that aid in facilitating the transition of young people with SCI from paediatric to adult health services.

2. Understand the experience of transition for young people with SCI and their parents/caregivers.

3. Explore the current needs of young people with SCI to identify gaps within the transition process.

4. Co-design and develop a HCT intervention to support young people with SCI.
5. Implement the HCT intervention and evaluate its acceptability and feasibility in supporting the transition process.

\section{Methodology}

This study draws on Article 7 of the United Nations Convention on the Rights of Persons with Disabilities (UNCRPD) ${ }^{14}$ and Article 12 of the United Nations Convention on the Rights of the Child (UNCRC) ${ }^{15}$ to inform its research methodology. The UNCRC acknowledges that children have the right to express their opinions and to have those opinions heard and acted on when appropriate, to be protected from abuse or exploitation and have their privacy protected. ${ }^{15}$ Article 7 of the UNCRPD furthers this sentiment specifically stating that actions should be taken to "ensure that children with disabilities have the right to express their views freely on all matters affecting them, their views being given due weight in accordance with their age and maturity, on an equal basis with other children, and to be provided with disability and age-appropriate assistance to realise that right'. ${ }^{14}$ As a result of the increase in emphasis on children's rights, the academic community have responded by ensuring children's participation in research on issues that affect them. ${ }^{16}$ Participatory action research (PAR) offers an approach to research that engages individuals and communities in identifying problems relevant to their own lives, redistributing the power between researcher and participants and giving them a chance to be part of social change. ${ }^{1617}$ The process champions the concept of 'research with, rather than on, people'. ${ }^{18}$

This study will be informed by PAR methodology as it seeks to understand the experience of young people with SCI, and focuses on equal and collaborative participation. Little has been written on the process of co-designing HCT interventions with young people with chronic conditions and disabilities (authors own work, currently under review). However despite the lack of literature on the development of HCT interventions using PAR and other co-design approaches, the authors reported that it was feasible to co-develop age-appropriate HCT interventions for young people with chronic conditions. Using a PAR framework to inform the methodology behind this study will ensure that the needs of young people with SCI are integral to the proposed interventional approach, and that their voice is heard and taken into consideration. The study will recognise and value the experiential knowledge of young people with SCI in understanding and addressing the key factors that impact on their successful transition from paediatric to adult healthcare services. Furthermore, it will integrate the input of young people in the design and implementation of the model, to secure their support of the HCT intervention.

\section{Theoretical framework}

This study will be informed in its thematic data analysis by the principles of critical disability theory (CDT). Critical theory is a multidisciplinary framework with a goal 
of explaining oppression and identifying achievable and practical ways to change it. ${ }^{19} \mathrm{CDT}$ centres disability and challenges ableist assumptions. Adopting CDT in this study will serve as a lens to examine transition needs and ensure the rights of children with disabilities are recognised while also respecting their voice, which has too often been marginalised.

\section{Contextual framework underpinning intervention development} and evaluation

This study will use the Care Transitions Framework to inform the development of the HCT intervention for young people with SCI transitioning from paediatric to adult healthcare services. The framework provides a guide to implementation, organised into eight domains; Intervention Characteristics, External Context, Organisational Characteristics, Characteristics and Roles of Providers, Characteristics and Roles of Patients and Caregivers, Process of Implementation, Measures of Implementation and Outcomes. ${ }^{20}$
A scoping review and ongoing consultations with services and stakeholders will generate knowledge on the external context, organisational characteristics and characteristics and roles of providers. Early engagement with health professionals, service providers and key stakeholders will provide an opportunity to understand the range of services available, the current gaps and some of the limitations to providing care. The phases of this study will address the remaining domains as outlined in figure 1.

\section{METHODS AND ANALYSIS}

There will be three phases to this study that will address the five objectives outlined in the Aims section of this paper. The phases and study objectives are depicted in figure 1 .

Phase 1 will include semi-structured individual interviews or paired interviews to explore current experiences

\section{Phase 1}

\section{Exploring current experiences} and unmet needs

\section{Care Transitions Framework Domain}

- Characteristics and Roles of Patients and Caregivers

\section{Study Objectives}

- Identify current services and resources that aid in facilitating the transition of young people with $\mathrm{SCl}$ from paediatric to adult health services.

- Understand the experience of transition for young people with $\mathrm{SCl}$ and their parents/caregivers.

- Explore the current needs of young people with $\mathrm{SCl}$ to identify gaps within the transition process.

\section{Phase 2}

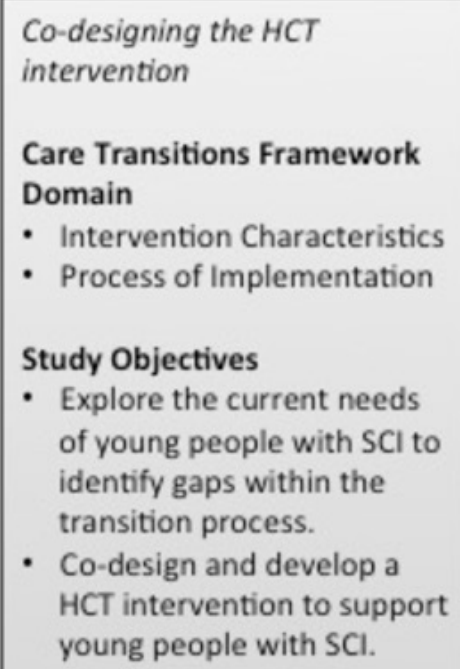

Co-designing the $\mathrm{HCT}$

intervention

\section{Care Transitions Framework} Domain

- Intervention Characteristics

- Process of Implementation

Study Objectives

- Explore the current needs of young people with $\mathrm{SCl}$ to identify gaps within the transition process.

- Co-design and develop a $\mathrm{HCT}$ intervention to support young people with $\mathrm{SCl}$.

\section{Phase 3}

Implementing and evaluating

the HCT intervention

\section{Care Transitions Framework}

Domain

- Measures of Implementation

- Outcomes

\section{Study Objectives}

- Implement the HCT intervention and evaluate its acceptability and feasibility in supporting the transition process. the domains of the care transition framework and how the study objectives within each phase address the domains. HCT, healthcare transition; $\mathrm{SCl}$, spinal cord injuries. 


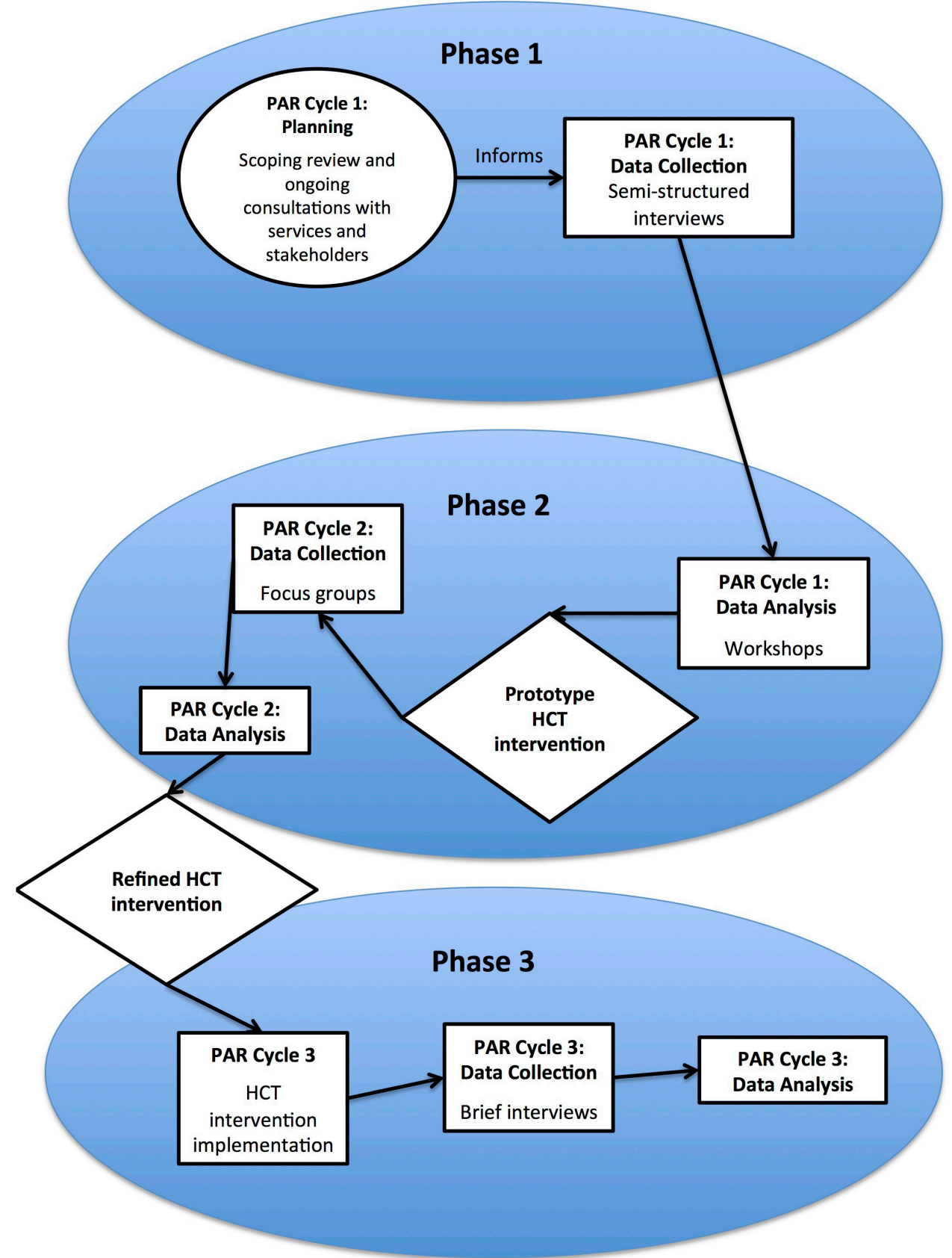

Figure 2 Participatory action research cycles and study phases. This figure depicts the cycles of the participatory action research methodology adopted in this study. HCT, healthcare transition; PAR, participatory action research.

and unmet needs. Phase 2a will use group co-design workshops to help analyse the interviews and inform the development of a prototype HCT intervention. Phase $2 \mathrm{~b}$ will gather feedback on the prototype HCT intervention from the young people and parents/caregivers involved in the workshops as well as the reference group to allow refinement and revision and improved practical application. Phase 3 will implement the HCT intervention and evaluate its acceptability and feasibility.

All phases of the study will be informed by the principles of PAR and involve cycles of planning, acting, observing and reflecting as described by Kemmis and McTaggart. ${ }^{21}$
Three PAR cycles will be conducted throughout the study as per figure 2 .

\section{Study setting}

Due to the small population of paediatric-onset SCI, ${ }^{22}$ the study will cover both metropolitan and rural New South Wales, Australia. It is a goal of this study to recognise the importance of providing opportunities for participants from rural areas to be involved in the study as young people with disabilities can be particularly disadvantaged in rural areas. ${ }^{23}$ Often they experience a lack of services and continuity of care, ${ }^{23}$ as such their experiences transitioning from paediatric to adult healthcare services may 
vary greatly from young people residing in metropolitan areas.

\section{Study population}

Young people between the ages of 14-25 years who acquired a paediatric-onset SCI (before the age of 16) and parents / caregivers of young people with a paediatriconset SCI will be eligible to participate. Young people will either be preparing to transition or will have transitioned. Individuals who are currently an inpatient in a children's hospital receiving rehabilitation treatment for a SCI acquired in the last 12 months will not be eligible for inclusion. The first year after injury can be overwhelming and requires tremendous adjustments for both the individual and their family. The researcher does not want to burden the individual or their family with the demands of participating in research, nor risk causing any additional emotional distress during this challenging time.

The study will also only include young people with SCI and parents/caregivers with sufficient English language proficiency to allow for engagement in discussions during the interviews and participation in the co-design workshop. Please note hereafter and unless otherwise specified, the term participants will be used to denote both young people with SCI and parents/caregivers.

\section{Recruitment and sample size}

The researcher will use purposive, convenience and snowball methods of recruitment for the study. An electronic flyer and video will be emailed to paediatric SCI support organisations for advertisement through their networks and on their social media pages and websites. The researchers will also recruit through word-of-mouth and social media (Twitter, LinkedIn, Facebook). Approximately six to eight young people with SCI and six to eight parents/caregivers of young people with SCI will be recruited for this study.

The researchers acknowledge that participants may drop-out between the different phases of the study. If this occurs, the researchers will attempt to recruit new participants as knowledge from previous phases, while helpful, is not required for inclusion in later phases.

\section{Patient and public involvement}

Young people with SCI and their parents/caregivers were not involved in setting the research question or design of the study, but they will be heavily involved in the design, implementation and evaluation of the HCT intervention.

A reference group, consisting of paediatric SCI healthcare service providers and young people with SCI specifically chosen for their particular areas of expertise, will be consulted throughout the study and asked to provide expert advice on:

- Recruitment;

- Appropriateness of the interview schedule and co-design workshop activities;

- Identification of issues or barriers that could impede the success of the study;
- Identifying solutions to problems with implementation of the study;

- Discussing the key outcomes of the study and;

- Providing feedback on the final HCT intervention.

\section{Phase 1: exploring current experiences and unmet needs}

Data collection

Semi-structured interviews will be used to collect qualitative data on the HCT experiences of young people with SCI from the perspectives of the young people themselves and parents/caregivers. Semi-structured interviews have been chosen to allow for the flexibility to explore ideas and responses that are important to participants but may not have been previously considered by the researchers. ${ }^{24}$ Semi-structured interviews will also allow for the researcher to adapt the interview techniques to the child or young person's developmental age.

In an attempt to reduce power imbalances, participants will be offered the opportunity to participate in a paired interview with another young person that is also eligible for the study. Paired interviews, where a pre-established relationship exists, can provide a more complete picture of the issues as the other interviewee supports the filling of gaps in the story. ${ }^{25}$

Interview guides will be developed to explore the needs, gaps, weaknesses and opportunities relating to HCT for young people with SCI. The interviews will be conducted online via the use of video conferencing software (Zoom) at a time that is convenient to the participant. The interviews are anticipated to take $60 \mathrm{~min}$. Interviews will be audio-recorded and transcribed verbatim to assist with data analysis.

\section{Analysis}

An inductive thematic analysis approach, as described by Braun and Clarke, ${ }^{26}{ }^{27}$ will be undertaken to identify major themes and subthemes arising from the participant responses. This style of analysis involves six phases: familiarisation, code generation, searching for themes, review and theme naming and report production. These phases need not be treated linearly and thus movement between the six phases will occur as required. An inductive thematic analysis approach has been chosen as it allows for a 'bottom up' analysis of the data to occur, whereby analysis is not driven by the researchers' preconceptions or pre-existing coding frame but instead is driven by the participants' responses and strongly links the themes identified to the data. ${ }^{27}$

PAR methodology requires collaboration at all stages of the research progress and Liebenberg $e t a t^{28}$ suggest that simply 'being reflexive and conducting member checks of findings from the analysis is insufficient'. Instead participants should actively participate in the data analysis; however, guidelines on how this can be successfully achieved is limited. ${ }^{28}$

In this study, the principal researcher and another member of the research team will individually code the transcripts. Following this, a final list from each reviewer 
will be developed and a meeting hosted, where through consensus, a final list of themes and subthemes will be determined. In addition to this, the co-design workshops will begin by asking the participants to review the codes and themes generated from the individual interviews. Researcher and participants (co-researchers) will compare and discuss the analysis decisions until consensus is achieved.

\section{Phase 2a: co-designing the HCT intervention (workshops) Data collection}

The co-design workshops will be facilitated by two researchers and are anticipated to take between 60 and $90 \mathrm{~min}$. The workshops will either be held in person at the university campus or via video conferencing (Zoom) at a time convenient for the group. There will also be the option for rural and remote participants to dial into the workshops using Zoom. Two workshops will be held-one for young people with SCI and one for parents/caregivers.

The workshops will have two phases, the first being to analyse the data from the interviews and the second to co-design the HCT intervention.

The first phase of the workshop will require participants to review samples of unidentifiable excerpts of interviews and initial codes/categories generated by the researchers and decide on their authenticity. Once in agreement on codes/categories, working together researchers and participants (co-researchers) will group codes/categories into themes using post-it notes or the white-board function on Zoom. During this process, participants (co-researchers) will be asked to explain their analysis decisions to the researchers.

In the co-design phase young people's HCT needs and participants' recommendations for the development of the HCT intervention will be explored. The researcher will use the future workshop method ${ }^{29} 30$ to facilitate discussion and generation of ideas for the development of the HCT intervention and participants will then work together to brainstorm designs. The future workshop method guides participants through three phases: a critique phase, a fantasy phase and an implementation phase. ${ }^{29}{ }^{30}$ The aim of the critique phase will be to review the themes identified in the data analysis phase to identify deficits or challenges related to HCT experienced by young people with SCI. In the fantasy phase, the participants will be given creative freedom to generate utopian ideas about the best possible way to mitigate the issues. In the third phase, the participants will transform the utopian ideas into a design for a practical and realisable HCT intervention.

\section{Analysis}

The workshops will be audio-recorded. The design ideas developed in the workshops will be captured in creative forms such as drawings/writings or verbally. Participants will also be asked to describe their design ideas to the group so that the researchers can capture these in the audio-recordings.
Phase 2b: co-designing the HCT intervention (focus groups) Data collection

In this phase participants will be provided/shown the prototype intervention and invited to partake in a focus group conducted online using video conferencing software (Zoom). Qualitative data will be collected to identify whether the HCT needs of young people with SCI and participants' recommendations have been met by the prototype. The focus groups are anticipated to take approximately $60 \mathrm{~min}$ and will be facilitated by two researchers. The focus group will be audio-recorded and transcribed to assist with data analysis.

\section{Analysis}

The transcripts from the focus groups will be analysed thematically in a similar process to Phase 1 in order to identify any adjustments required to the HCT intervention. The principal researcher and another member of the research team will individually code the transcripts. A final list from each reviewer will be developed and a meeting hosted, where through consensus, a final list of themes and subthemes will be determined and the HCT intervention will be refined based on this feedback.

\section{Phase 3: implementing and evaluating the HCT intervention} Data collection

Due to the iterative nature of PAR, it is anticipated that the HCT intervention will evolve within the research process as participants' experiences and needs influence its development. As such, the nature of the final HCT intervention cannot be known prior to the commencement of the study. Nevertheless, following the completion of the focus groups the researcher will refine the prototype HCT intervention and send the final HCT intervention to participants. They will be asked to review or use the HCT intervention and will be invited to partake in a brief interview. Brief interviews will be conducted over the telephone or online using video conferencing software (Zoom) at a time that is convenient to the participant. The interviews are anticipated to take between 15 and $20 \mathrm{~min}$.

The interviews will evaluate the feasibility and acceptability of the HCT intervention. Bowen and colleagues ${ }^{31}$ framework will inform the evaluation of the HCT intervention (online supplemental file 1). This framework will support making judgements about the feasibility of the intervention and determine whether additional, more comprehensive evaluation is justified. As this study is part of a 3-year doctoral project, time constraints restrict the researchers on conducting a comprehensive pilot study of the HCT intervention to determine its efficacy and effectiveness. Using Bowen and colleagues' framework the researchers will be able to determine whether the HCT intervention is appropriate for further testing, and is relevant and sustainable.

Analysis

Data analysis of Phase 3 will mirror the analysis method in the earlier phases. 


\section{Rigour}

To ensure that the rigour of the study's qualitative data is maintained the researcher will address the following criteria: credibility, transferability, dependability and confirmability. ${ }^{32}$ In regard to credibility, the researchers will engage in frequent debriefing sessions to provide an opportunity for the researchers to identify and challenge any assumptions made as a result of their own biases and preferences. Furthermore, credibility will be achieved by including young people with SCI and their parents/caregivers as co-researchers in the analysis of the interviews and by providing opportunities for them to review, reflect on and refine the co-developed HCT intervention.

Transferability will be achieved through detailed reports, thick descriptions and analysis of contextual details, as described by Ponterotto. ${ }^{33}$ Such details include demographic information, the location and settings of the interviews, workshops and focus groups and descriptions about non-verbal behaviour. The researchers acknowledge that the study findings and the tailored intervention may not be applicable to populations outside the study setting. Therefore, a comprehensive description of the process involved in co-designing a HCT intervention with young people with lived experience of SCI will be provided, as the co-design process may have transferability across contexts and medical conditions.

The researcher will maintain an audit trail and report in detail the processes that occurred within the study related to research design and implementation, operational details of data gathering and provide a reflective appraisal of the processes undertaken. This process will enhance dependability.

Lastly throughout the project, the researcher will keep a comprehensive reflective research journal, reflecting on and cataloguing the progress, obstacles and successes of the research process. This level of documentation will increase confirmability of the research by providing an audit trail for the study.

\section{Ethical and safety issues}

This study has received ethics approval from the Western Sydney University Human Research and Ethics Committee (H14029).

Written consent will be required from all participants prior to their involvement in the study (online supplemental file 2). As the study includes young people under the age of 16 years, the study aims, objectives and requirements will be discussed with these individuals using ageappropriate language and written consent will also be required from their parent/caregiver. Verbal consent will also be obtained from all participants at the beginning of each interview, workshop or focus group prior to starting any recordings.

Given the small size of the paediatric-onset SCI community in which this study will be undertaken, there are ethical considerations in relation to protecting the anonymity of participants and confidentiality of data, particularly regarding workshops and focus groups. Before starting any group activities, participants will be advised that all personal information shared in the discussion will be kept confidential and is not for discussion outside of the group. Participants will be asked to uphold the principle of respect regarding their own behaviour and the privacy of other participants. Additionally, the utmost care will be taken in analysis and presentation of data to ensure participant confidentiality and anonymity. Data that may overtly identify participants will be excluded.

It is possible that recalling experiences associated with acquiring an SCI or negative experiences with healthcare services may cause some discomfort to the young people or parents/caregivers. As such, the researchers will monitor and respond to participant's psychological wellbeing. Information on where to access emotional support will be made available to all participants.

\section{Dissemination}

As this is a supervised doctoral research study the researcher will use the results as chapters of a thesis to obtain a Doctor of Philosophy degree. It is anticipated that the findings from this study will also be disseminated via publication in peer-reviewed journals and will be presented at local, national or international conferences and professional forums. Participants will also be invited to co-present with the researcher at a local conference or professional forum. The progress and findings of the study will be communicated to young people with SCI and parents/caregivers as well as professional stakeholders via reports and websites maintained by SCI organisations, as well as through social media.

\section{Twitter Emily Alice Bray @Emily_A_Bray}

Acknowledgements The authors would like to acknowledge SpineCare Foundation for funding this study and for their continued support.

Contributors $E A B, B E, A G, Y S$ and $L R$ were responsible for the study conception and design. $E A B, B E, A G, Y S$ and $L R$ were responsible for drafting the manuscript and making critical revisions to the paper for important intellectual content.

Funding This work was supported by the SpineCare Foundation, Sydney, Australia. Grant number: N/A.

Competing interests None declared.

Patient consent for publication Not required.

Provenance and peer review Not commissioned; externally peer reviewed.

Supplemental material This content has been supplied by the author(s). It has not been vetted by BMJ Publishing Group Limited (BMJ) and may not have been peer-reviewed. Any opinions or recommendations discussed are solely those of the author(s) and are not endorsed by BMJ. BMJ disclaims all liability and responsibility arising from any reliance placed on the content. Where the content includes any translated material, BMJ does not warrant the accuracy and reliability of the translations (including but not limited to local regulations, clinical guidelines, terminology, drug names and drug dosages), and is not responsible for any error and/or omissions arising from translation and adaptation or otherwise.

Open access This is an open access article distributed in accordance with the Creative Commons Attribution Non Commercial (CC BY-NC 4.0) license, which permits others to distribute, remix, adapt, build upon this work non-commercially, and license their derivative works on different terms, provided the original work is properly cited, appropriate credit is given, any changes made indicated, and the use is non-commercial. See: http://creativecommons.org/licenses/by-nc/4.0/.

ORCID iDs

Emily Alice Bray http://orcid.org/0000-0002-6069-6156 
Ajesh George http://orcid.org/0000-0002-6795-2546

Bronwyn Everett http://orcid.org/0000-0003-1733-7462

Yenna Salamonson http://orcid.org/0000-0002-7429-4086

Lucie Ramjan http://orcid.org/0000-0001-7815-3005

\section{REFERENCES}

1 Blum RW, Garell D, Hodgman $\mathrm{CH}$. Transition from child-centered to adult health-care systems for adolescents with chronic conditions: a position paper of the Society for adolescent medicine. Journal of Adolescent Health 1993;14:570-6.

2 Kerr H, Price J, Nicholl H, et al. Facilitating transition from children's to adult services for young adults with life-limiting conditions (TASYL): programme theory developed from a mixed methods realist evaluation. Int J Nurs Stud 2018;86:125-38.

3 Acuña Mora M, Saarijärvi M, Moons P, et al. The scope of research on transfer and transition in young persons with chronic conditions. $J$ Adolesc Health 2019;65:581-9.

4 Tsybina I, Kingsnorth S, Maxwell J, et al. Longitudinal evaluation of transition services ("LETS Study"): protocol for outcome evaluation. BMC Pediatr 2012;12:1-11.

5 Gray WN, Schaefer MR, Resmini-Rawlinson A, et al. Barriers to transition from pediatric to adult care: a systematic review. J Pediatr Psychol 2018;43:488-502.

6 Reiss J, Gibson R. Health care transition: destinations unknown. Pediatrics 2002;110:1307-14.

7 Wiemann CM, Graham SC, Garland BH, et al. In-Depth interviews to assess the relevancy and fit of a peer-mentored intervention for transition-age youth with chronic medical conditions. J Pediatr Nurs 2020;50:121-7.

8 Gabriel P, McManus M, Rogers K, et al. Outcome evidence for structured pediatric to adult health care transition interventions: a systematic review. J Pediatr 2017;188:263-9.

9 Rupp R, Biering-Sørensen F, Burns SP, et al. International standards for neurological classification of spinal cord injury: revised 2019. Top Spinal Cord Inj Rehabil 2021;27:1-22.

10 Zebracki K, Anderson C, Chlan K, et al. Outcomes of adults with pediatric-onset spinal cord injury: longitudinal findings and implications on transition to adulthood. Top Spinal Cord Inj Rehabil 2010;16:17-25.

11 Hwang M, Zebracki K, Vogel LC, et al. Development of the pediatric quality of life Inventory ${ }^{\mathrm{TM}}$ spinal cord injury (PedsQL ${ }^{\mathrm{TM}} \mathrm{SCI}$ ) module: qualitative methods. Spinal Cord 2020;58:1134-42.

12 Baker I, de Paula A, Serratore L, et al. Towards independence: the new South Wales (Australia) experience of transition to adulthood of young people with spinal cord injury. Top Spinal Cord Inj Rehabil 2010;16:55-65.

13 Botchway EN, Knight S, Muscara F, et al. Rehabilitation models of care for children and youth living with traumatic brain and/or spinal cord injuries: a focus on family-centred care, psychosocial wellbeing, and transitions. Neuropsychol Rehabil 2020:1-23.
14 United Nations General Assembly. Convention on the rights of persons with disabilities. Treaty Series 2006;2515:3

15 United Nations General Assembly. Convention on the rights of the child. United Nations, Treaty Series 1989;1577:1-23.

16 Shamrova DP, Cummings CE. Participatory action research (PAR) with children and youth: an integrative review of methodology and PAR outcomes for participants, organizations, and communities. Child Youth Serv Rev 2017;81:400-12.

17 Ozer EJ. Youth-Led participatory action research: developmental and equity perspectives. Adv Child Dev Behav 2016;50:189-207.

18 Savin-Baden M, Wimpenny K. Exploring and implementing participatory action research. Journal of Geography in Higher Education 2007;31:331-43.

19 Hosking DL. Critical disability theory. A paper presented at the 4th biennial disability studies conference at Lancaster University, UK. Journal of Consulting and Clinical Psychology 2008;72:467-78.

20 Rojas Smith L, Ashok M, Morss Dy S, et al. Contextual frameworks for research on the implementation of complex system interventions. Rockville (MD): Agency for Healthcare Research and Quality (US), 2014.

21 Kemmis S, McTaggart R. The action research planner. Victoria, Australia: Deakin University, 1988.

22 Parent S, Mac-Thiong J-M, Roy-Beaudry M, et al. Spinal cord injury in the pediatric population: a systematic review of the literature. $J$ Neurotrauma 2011;28:1515-24.

23 Balsamo F. Education access: national inquiry into rural and remote education, 2000. http://www.hreoc.gov.au/human_rights/rural/ education/index

24 Gill P, Stewart K, Treasure E, et al. Methods of data collection in qualitative research: interviews and focus groups. Br Dent $J$ 2008;204:291-5.

25 Wilson AD, Onwuegbuzie AJ, Manning LP. Using paired depth interviews to collect qualitative data. Qualitative Report 2016;21.

26 Braun V, Clarke V. Reflecting on reflexive thematic analysis. Qualitative Research in Sport, Exercise and Health 2019;11:589-97.

27 Clarke V, Braun V, Terry G. Thematic analysis. In: Liamputtong P, ed. Handbook of research methods in health and social sciences. Singapore: Springer, 2019: 843-60.

28 Liebenberg L, Jamal A, Ikeda J. Extending youth voices in a participatory thematic analysis approach. Int J Qual Methods 2020;19:160940692093461.

29 Alminde $\mathrm{S}$, Warming $\mathrm{H}$. Future workshops as a means to Democratic inclusive and empowering research with children, young people and others. Qualitative Research 2020;20:432-48.

30 Müllert N, Jungk R. Future workshops: how to create desirable futures. London, United Kingdom: Institute for Social Inventions, 1987.

31 Bowen DJ, Kreuter M, Spring B, et al. How we design feasibility studies. Am J Prev Med 2009;36:452-7.

32 Shenton AK. Strategies for ensuring trustworthiness in qualitative research projects. Education for Information 2004;22:63-75.

33 Ponterotto JG. Brief note on the origins, evolution, and meaning of the qualitative research concept "thick description". The Qualitative Report 2006;11:538+. 